\title{
Probabilistic Characterization of Current Harmonics of Electrical Traction Power Supply System by Analytic Method
}

\author{
YE Zhongming, LO Edward*, IEEE member \\ YUEN K. H.*, IEEE student member, PONG M. H*, IEEE senior member \\ *Dept. of Electrical \& Electronics Engineering \\ Hong Kong University, Pokfulam Road, Hong Kong, PRC \\ Nanjing Huaneng Electric Power Co. Ltd \\ Nanjing, Jiangsu, P. R. CHINA
}

\begin{abstract}
Current harmonics of an urban railway traction system in Hong Kong are investigated by analytic approach. With the statistic knowledge of speed and notch number of the trains in the system, the mean and variance of harmonic current of individual trains are computed based on a behavior oriented model of the traction electrical unit. According to the Large Number Law and Central Limit Theorem, current harmonics at a substation, which is a vectorial summation of harmonics of random number of trains electrically connected to it, are normally distributed provided that the number of trains is large enough. A set of equations are established by which probability density functions (pdfs) of current harmonics at a substation are calculated and found to be in good accordance with results obtained by Monte Carlo simulation. This approach of harmonics evaluation for traction system with random loading has evident advantages of cheap, fast and convenience and with no compromise of accuracy.
\end{abstract}

Keywords: Harmonics; Traction system; Monte Carlo simulation

\section{INTRODUCTION}

The electrified urban railway system being studied in this paper is a $25 \mathrm{KV}, 50 \mathrm{~Hz}$ single-phase current collection catenary system being fed from two track-side feeder stations. In each feeder station, a single-phase power source is taken from local power distribution system via $132 / 25 \mathrm{KV}$ transformers connected in open-delta. It is then fed into a section of the catenary system and energize the trains. On board transformers installed at the bottom of the trains receive power from the overhead line through pantographs. Phasecontrolled thyristor electrical units convert the secondary output of the transformer into DC and power up the traction motors. In each such unit, two identical rectifier bridges are connected in series which are in turn connected to two secondary windings of the transformer. Motor current is controlled by firing angles of the thyristors. By firing different thyristors four notches are made available to control the speed of the traction motors. For notch 1 and 2 only the upper rectifier is put into operation, while for notch 3 and 4 , the lower rectifier is also put into operation. These four notches correspond to equivalent circuits shown in Fig. 1,2. The maximum speeds that each notches can reach are shown in Table I.
Table I

The properties of notches of the traction electrical unit in this paper

\begin{tabular}{|c|c|c|}
\hline Notch number & $\begin{array}{c}\text { Maximum speed } \\
(\mathrm{Km} / \mathrm{hr})\end{array}$ & Equivalent circuit \\
\hline 1 & 20 & Fig. 1 \\
\hline 2 & 40 & Fig. 1 \\
\hline 3 & 60 & Fig. 2 \\
\hline 4 & 120 & Fig. 2 \\
\hline
\end{tabular}

An urban railway system is a large scale consumer of electricity and its power quality is always a major concern. In this system, the phase controlled converter draw harmonic currents from the power grid during train motoring and braking. Harmonic current propagates through the electrical system of the traction network and may cause additional thermal losses on the overhead lines and transformers, it may also cause electromagnetic interference and potential overvoltage or over-current. The adverse effects of harmonics in the railway system and power system call for serious concern to both the train operation and the local utility authorities. As this traction system is expected to include more and more trains to meet increasing load in the near future, accurate prediction of harmonic contents become very important for the local railway company.

The stochastic nature of the loading of the traction system makes probabilistic knowledge of the harmonics of the system more valuable. Previous work has shown that Monte Carlo simulation is a very good tool for the evaluation of harmonics of a cluster of random power electronics loads with certain parameters being prescribed with some well known probability distributions, such as electric vehicle battery chargers and traction [1] [2] [3]. However intensive computation is an obvious drawback. In the 1990 s, some efforts were made to predict random system harmonics by analytic methods, for example, to predict the harmonic voltage level of a number of nonlinear loads [7][8][9][10]. This method directly calculates the statistics of harmonics with probability theory. Although it does not require much computation, the probability density function of load parameters and the relation between harmonics and parameters of nonlinear loads have to be available and mathematically expressible.

For many practical traction systems, mathematical relation between harmonics and load parameters can not be easily established owing to the complexity of the real system or lack of knowledge of details of the loads and control method. 
Often the probability features of load parameters are not simple enough to be represented by an existing probability function.

This paper presents a statistic evaluation of the harmonic current level of a railway traction system. It does not need an exact model of the power converter circuit of the trains, and yet it greatly reduces the amount of computation required compared to other statistical methods. Firstly, the traction electrical unit model is proposed by Behavior Model Technique (BMT). Secondly the stochastic behavior of the trains is studied and probabilistically characterized. Thirdly the harmonics expectation of a single electrical unit as well as a group of trains are presented based on the model of the traction electrical unit and the probability density function of speed of trains. Finally comparison of results between this method and Monte Carlo simulation are made to verify its validity.
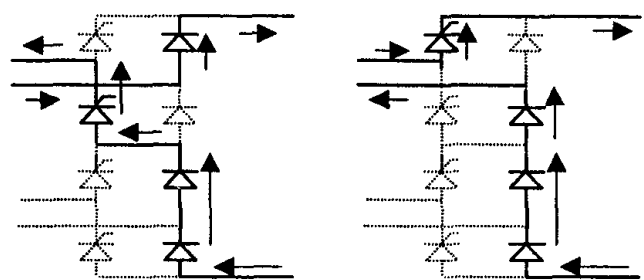

Fig. 1. Equivalent circuit while notch 1 and 2 working
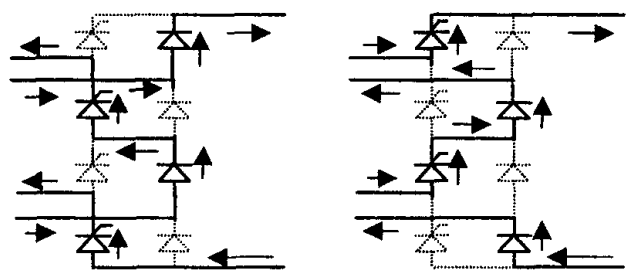

Fig. 2. Equivalent circuit while notch 3 and 4 working

\section{MODELING OF A TRACTION ELECTRICAL} UNIT

A traction elestrical unit consists of many different loads on board and the total current drawn is a complex combination of these equipment. Phase controlled thyristor rectifier with DC drive load, air-conditioning, lighting and other electrical equipment make up the load of the traction electrical unit. The complexity of the practical converter and load circuit makes simulation difficult and mathematical representation of the relation between harmonics and load parameters by equations is almost impossible. However, most current is drawn by the propulsion converter which is mainly determined by the operation modes of the train. Harmonic modelling of the electrical unit can be set up in a much easier way using behavior model technique.

The traction electrical unit concerned is treated as a black box [10], the input of which is the behavior state of traction, that is, the operation mode including speed and notch number $(v, n)$. For each such input, corresponding harmonics will be generated as output. By this method, one can determine the output harmonics from the input behavior state without detail knowledge of the traction electrical unit, and a realistic traction electrical unit can be simulated.

In order to establish a map between output harmonics and input behavior states of such a black box, experimental measurement of the practical traction electrical unit is necessary. An important input parameter is speed during motoring. Obviously, since speed is continuous from zero to maximum, to do all experiments for all these behavior states is impossible and unnecessary. Therefore the behavior state is sampled at discrete points. Tests for train characteristic are performed at these discrete states on a typical train which has typical electrical structure and similar working condition as other trains. The current waveforms of the traction electrical unit are recorded and converted to frequency domain by Fast Fourier Transform Algorithm. For each order of harmonics, it can be represented as two dimensional variables in realimaginary projections:

$$
\begin{gathered}
Y_{h}(v, n)=X_{h}(v, n)+j Y_{h}(v, n) \\
\quad \text { for } h=1,2,3, \ldots \\
X_{h}=X_{h}(v, n) \\
Y_{h}=Y_{h}(v, n)
\end{gathered}
$$

where $X_{h}, Y_{h}$ are the real and imaginary part of the $h$ th harmonic components and $v, n$ stand for speed and notch number respectively.

In order to simulate the practical system, continuous representation of harmonics for all possible speeds are necessary. This can be done by interpolation of harmonics spectrum for various behavior states in frequency domain. By interpolating a map between the speed and harmonic components of current is established. Fig. 3 shows typical curves of amplitude and phase angle of fundamental, $3^{\text {rd }}, 5^{\text {th }}$, $7^{\text {th }}$ harmonics components versus speed during train motoring. The amplitude of the harmonics and fundamental currents are small at both low speeds and high speed, while different order of harmonics reach their maximum amplitudes at different speeds. The phase angles of individual harmonics components also change with speed.

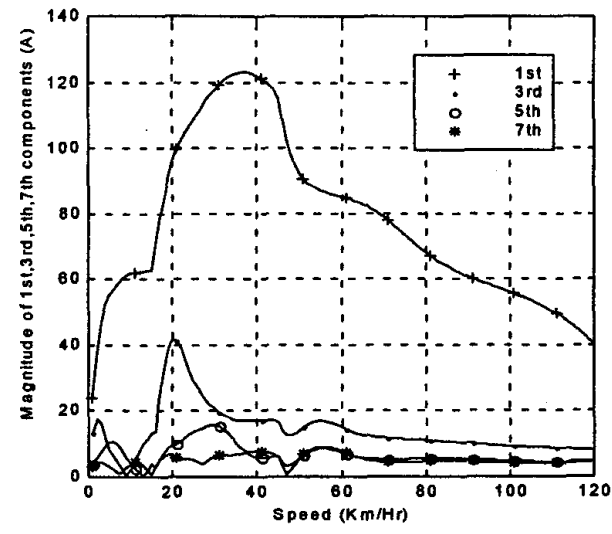

Fig. 3. The fundamental and 3,5,7 order harmonic current versus speed during acceleration (from top: 1 st, 3rd, 5th, 7th) 


\section{SPEED PROBABILITY DENSITY FUNCTION}

Behavior state of train including speed and notch number, which determines the current harmonics of traction electrical units, randomly changes in practical railway system. Probabilistic characterization of speed and notch number is a necessity for the prediction of the sum of randomly varying harmonics of the same order of both system level and individual trains. Either probability density function or cumulative probability density function is required.

In order to obtain the probabilistic features of a practical railway system, an automatic data logging system is developed and implemented with a notebook computer, data acquisition card and sensors. This equipment is installed on board a train in service. For every half second, it measures the speed signal of the train and for every half an hour it saves the recorded data on the hard disc of the computer. With a measurement lasting for about two weeks, about 600,000 samples are recorded. The harmonic current of the electrical train unit is also sampled.

Probability density function of speed is obtained from experimental data. According to probability theory, probability of an event can be approximated by the frequency of occurrence of the event. The error will be minimum if the number of samples is sufficiently large. In this way, the probability density function of speed of trains during motoring is obtained and shown below in Fig. 4. The speed increment per step of the measurement is $0.5 \mathrm{Km}$ per hour. The four traces in Fig. 4 are PDFs of speed during motoring for notch 1, 2, 3 and 4 accordingly. The top curve stands for the PDF of the speed for motoring, and is a sum of the individual PDFs of notch 1, 2,3 and 4. Thus the area under this curve should be unity. It can be seen that notch 4 are more frequently used at high speed than at low speed, while the usage of notch 1 and 2 are always at low speed. In addition, the probability of using notch 4 is much higher than other notches. Samples of speed at motoring occupy about $50 \%$ of the total samples for all operation modes, including speed samples while the train is coasting and braking.

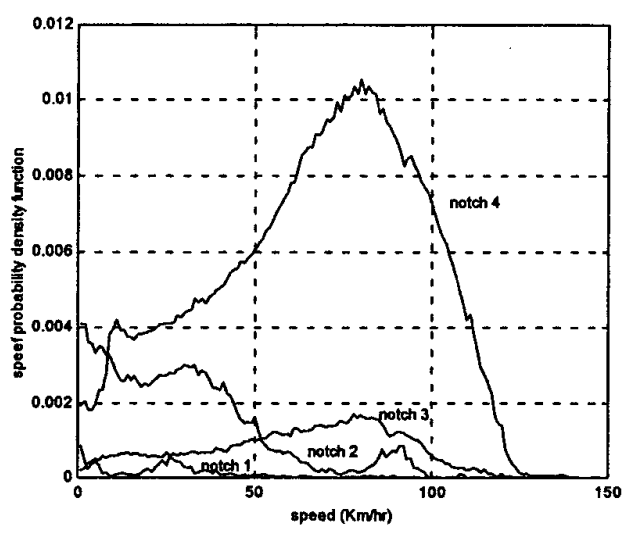

Fig. 4. The probability density function of speed for motoring operation only (from bottom to next to top: notch 1,2,3,4).
The probability density function of speed can be smoothed and then made discrete with a fixed interval $\Delta v=1 \mathrm{Km} / \mathrm{hr}$ to facilitate the following numerical calculation.

$p_{M n}$ denotes the probability density for speed ${ }^{v}$ with notch number ${ }^{n}$. The probability of speed $v_{i}$ with notch number $n j$ is defined as:

$$
P_{v_{i}, n_{j}}=P\left(v_{l} \leq V<v_{l+1}, \text { notch }=n_{j}\right)
$$

then it can be calculated from the probability density function Pn:

$$
P_{v_{i} n_{j}}=\frac{\left(p_{r_{i} \eta_{j}}+p_{v_{i+1} n_{j}}\right)}{2} \Delta v
$$

\section{HARMONIC CURRENT OF INDIVIDUAL TRACTION ELECTRIC UNIT}

As the probabilistic knowledge of speed is obtained, current harmonics of individual train can be predicted with the help of the traction electrical unit model. Statistical method like Monte Carlo method can be used for the prediction but it will take days of computation time on a PC. A more direct calculation method is proposed which can drastically cut down computation time. Equations are established directly from probabilities of speed and notch, together with corresponding harmonic current.

The mean of the real part of harmonics with order $h$ of a single electrical unit can be evaluated from the following equations:

$$
\begin{aligned}
\overline{\mu_{x_{n}}} & =\sum_{\text {notch }}^{4} \int X_{n}(v, n) p_{v n} d v \\
& =\sum_{\text {notch }}^{4} \sum_{v=0}^{\text {Moxspeed }} X_{n}(v, n) P_{v n}
\end{aligned}
$$

The variance of the real part of the current harmonics of order $\mathrm{h}$ can be calculated from:

$$
\overline{\sigma X_{h}}=\sqrt{\sum_{\text {notch }}^{4} \sum_{\nu=0}^{\text {Mocspeed }} X h(v, n)^{2} P_{\nu, n}-\left(\overline{\mu_{x_{h}}}\right)^{2}}
$$

Similarly, one can get the formula to calculate the mean of the imaginary part:

$\overline{u Y_{n}}=\sum_{\text {notch }}^{4} \sum_{\nu=0}^{\text {Moxspeed }} Y_{h}(v, n) P_{v n}$

and the variance of the imaginary part of current harmonics

$$
\widetilde{\sigma Y_{h}}=\sqrt{\sum_{n \text { otch }}^{4} \sum_{v=0}^{\text {Maxspeed }} Y_{h}(v, n)^{2} P_{v, n}-\left(\overline{\mu_{Y_{h}}}\right)^{2}}
$$


In order to verify results obtained by this method Monte Carlo simulation is carried out with 30,000 samples. The mean and variance of current harmonics up to the 19th order for individual train electric unit during motoring are calculated and compared in Table II with the ones obtained from Monte Carlo simulation. Good agreement are observed between these two approaches. The mean and variance of experimental data of fundamental current, 3rd, 5th, 7th harmonics for two weeks are then calculated and compared with the above results which shows approximately $10 \%$ error. From the point of engineering view, this error is acceptable.

In table III, the mean and variance of current harmonics components for different notches are given. From the table, it is evident when notch 1 and 2 are used, the expected harmonic current of the electrical unit is low in magnitude, however, the waveform distortion is very serious. By contrast, the expected harmonics current for notch 3 and 4 are large in magnitude, but the waveform distortion is very small.

Table II

The Mean \& Variance of Current Harmonics of Individual Electric Unit During Motoring (UNIT : A)

$$
\text { I - Analytic method II - simulation }
$$

\begin{tabular}{|c|c|c|c|c|c|c|c|c|c|}
\hline & \multicolumn{3}{|c|}{ magnitude of harmonics } & \multirow{2}{*}{\multicolumn{3}{|c|}{ real part of current }} & \multicolumn{3}{|c|}{ imaginary part current } \\
\hline & & $\operatorname{can}$ & var & & & & & $\operatorname{san}$ & var \\
\hline nethod & I & II & 1 & 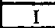 & II & I & I & II & I \\
\hline $1^{n}$ & 70.92 & 70.75 & 28.68 & 57.58 & 57.33 & 28.69 & 38.93 & 38.98 & 14.08 \\
\hline $3^{\text {nt }}$ & 12.36 & 12.31 & 6.6 & 8.25 & 8.21 & 6.74 & 5.14 & 5.26 & 7.51 \\
\hline $5^{\text {th }}$ & 5.66 & 5.64 & 2.99 & 1.49 & 1.46 & 3.92 & 3.39 & 3.43 & 3.45 \\
\hline $7^{\text {th }}$ & 4.44 & 4.46 & 2.12 & -1.1 & -1.1 & 3.66 & 2.33 & 2.34 & 2.47 \\
\hline $9^{\text {th }}$ & 3.69 & 3.71 & 1.46 & -2.1 & -2.14 & 3.07 & -0.36 & 0.36 & 1.34 \\
\hline $11^{\#}$ & 2.62 & 2.62 & 1.01 & 0 & 0 & 1.56 & 1.59 & 1.59 & 1.7 \\
\hline $13^{\text {nd }}$ & 1.53 & 1.53 & 0.66 & 0.55 & 0.55 & 0.99 & 0.2 & 0.19 & 1.21 \\
\hline
\end{tabular}

Table III

Mean and variance of harmonicg during motoring for notch $1,2,3,4$ respectively (unit: A)

\begin{tabular}{|c|l|l|l|l|l|l|l|l|}
\hline & \multicolumn{2}{|c|}{ notch 1 } & \multicolumn{2}{c|}{ notch 2 } & \multicolumn{2}{c|}{ notch 3 } & \multicolumn{2}{c|}{ notch 4 } \\
\hline & mean & var & mean & var & mean & var & mean & var \\
\hline 1 & 5.45 & 7.06 & 32.66 & 22.2 & 93.76 & 14.47 & 77.04 & 22.27 \\
\hline 3 & 3.8 & 4.39 & 6.41 & 4.52 & 15.28 & 5.66 & 13.37 & 6.29 \\
\hline 5 & 1.41 & 1.17 & 2.86 & 2.79 & 6.27 & 2.64 & 6.25 & 2.68 \\
\hline 7 & 0.65 & 0.98 & 1.24 & 1.39 & 5.23 & 1.18 & 5.08 & 1.61 \\
\hline 9 & 1.04 & 1.09 & 2.1 & 1.41 & 4.39 & 0.83 & 3.99 & 1.25 \\
\hline 11 & 0.43 & 0.33 & 2.03 & 1.6 & 2.84 & 0.68 & 2.75 & 0.77 \\
\hline 13 & 0.46 & 0.55 & 1.73 & 1.1 & 1.66 & 0.45 & 1.49 & 0.52 \\
\hline 15 & 0.46 & 0.48 & 1.3 & 0.88 & 1.13 & 0.24 & 0.85 & 0.42 \\
\hline 17 & 0.27 & 0.28 & 1.08 & 0.72 & 0.79 & 0.27 & 0.82 & 0.24 \\
\hline 19 & 0.47 & 0.36 & 0.85 & 0.43 & 0.68 & 0.26 & 0.79 & 0.26 \\
\hline
\end{tabular}

Table IV

Expected mean and variance of harmonics for moving trains (unit: A)

\begin{tabular}{|l|c|c|c|c|c|c|c|c|c|c|}
\hline & ist & 3rd & 5th & th & 9th & 11 st & 13 rd & 15 th & 17 th & 19 th \\
\hline mean & 24 & 4.8 & 2.3 & 1.6 & 1.4 & 0.9 & 0.5 & 0.3 & 0.3 & 0.4 \\
\hline variance & 38.2 & 6.8 & 3.13 & 2.49 & .84 & 1.26 & 0.71 & 0.43 & 0.38 & 0.29 \\
\hline
\end{tabular}

In Table IV, the expected mean harmonics components for all operation modes, including motoring, coasting and braking are presented. It can be seen from the table that the expected value of harmonics for a moving train is very low, $35 \%$ of the values for motoring, that is because for more than $50 \%$ possibility the electrical unit of trains use notch zero, that is, generate very slight harmonic current. The difference of expected values for harmonic distortion between the case of motoring and moving (including all operation modes) is small.

\section{HARMONICS CURRENT OF N TRAINS}

A major concern is harmonic currents at a substation which feeds a number of trains. When $\mathrm{N}$ trains in a section are considered, the current at the substation can be approximated by the vectorial summation of the harmonics phasors of $\mathrm{N}$ trains in frequency domain for each order. In urban railway a section is usually short and the one being consider is less than $20 \mathrm{~km}$ and the impedance of the power line is very small thus can be ignored. The harmonic current of order $h$ is represented by :

$$
\Gamma_{h}=\sum_{j=1}^{N} X_{j h}+j \sum_{j=1}^{N} Y_{j h} \text { for } h=1,2,3, \ldots
$$

$$
\begin{aligned}
& \text { Denote: } \\
& S_{X_{h}}=\sum_{j=1}^{N} X_{j, h} \quad S_{Y_{h}}=\sum_{j=1}^{N} Y_{j, h}
\end{aligned}
$$

Assume the behavior states of $\mathrm{N}$ trains are independent of one another and only moving trains in the system are considered. The PDF of the sum $S X_{h}$ is the standard convolution integral of the individual probability density function of the real part of the harmonics components:

$$
p s_{x_{h}}=p_{1, X_{h}} * p_{2, X_{h}} * \ldots * p_{N, X_{h}}
$$

where $p_{1}, X_{n}, p_{2}, X_{n}, \ldots, p_{N} X_{k}$ stand for the probability density function of individuals and the asterisk stand for convolution integral operator.

According to the Large Number Law and Central Limit Theorem, the distribution of the current sum will approximate normal distribution as long as $\mathrm{N}$ here is sufficiently large. In a real situation, this is also true even for a comparatively small $\mathbf{N}[6]$ [8].

Therefore, the probability density function of the sum can be approximated by a normal distribution as:

$$
f_{S_{X_{h}}}\left(S_{X_{h}}\right)=\frac{\left(\frac{-\left(s_{X_{h} * s_{X_{h}}}\right)^{2}}{2 \sigma_{S_{X_{h}}}^{2}}\right)}{\sqrt{2 \pi} \sigma_{S_{X_{h}}}^{2}}
$$

Similarly,

$$
f_{S_{Y_{h}}}\left(S_{Y_{h}}\right)=\frac{\left(\frac{-\left(S_{Y_{h}}+S_{Y_{Y_{h}}}\right)^{2}}{2 \sigma \sigma_{Y_{h}}}\right)}{\sqrt{2 \pi} \sigma_{S_{Y_{h}}}^{2}}
$$

In the above, the mean and variance of the sum $S_{X}, S_{Y}$ are the sum of mean and variance of individuals: 


$$
\begin{aligned}
u_{S_{X_{h}}}=\sum_{j=1}^{N} \mu_{i, X_{h}} & u_{S_{Y_{h}}}=\sum_{i=1}^{N} \mu_{i, Y_{h}} \\
\sigma_{S_{X_{h}}}^{2}=\sum_{j=1}^{N} \sigma_{i, X_{h}}^{2} ; & \sigma_{S_{Y_{h}}}^{2}=\sum_{j=1}^{N} \sigma_{i, Y_{h}}^{2}
\end{aligned}
$$

Since all trains are assumed to have the same probabilistic distribution, the mean and variance of $S_{X}, S Y_{\text {can }}$ be calculated:

$$
\begin{gathered}
u_{S_{X_{h}}}=N \mu_{X_{h}} u_{S_{Y_{h}}}=N \mu Y_{h} \\
\sigma_{S_{X_{h}}}^{2}=N \sigma_{X_{h}}^{2} ; \quad \sigma_{S_{Y_{h}}^{2}}^{2}=N \sigma_{Y_{h}}^{2}
\end{gathered}
$$

The joint probability density function of the sum of real part and sum of the imaginary part can be calculated:

$$
f_{S_{X_{h}} S_{Y_{h}}}\left(S_{X_{h}}, S_{Y_{h}}\right)=\frac{1}{2 \pi \sigma_{S_{X_{h}}} \sigma_{S_{Y_{h}}} \sqrt{1-p^{2}}}
$$

where the correlation coefficient is defined as:

and

$$
\begin{gathered}
\rho=\frac{\sigma_{S_{X_{h}}}^{2} s_{Y_{h}}}{\sigma s_{X_{h}} \sigma s_{Y_{h}}} \\
\sigma_{S_{X_{h}} S_{Y_{h}}}=N \sigma_{X_{h} Y_{h}}^{2}
\end{gathered}
$$

$$
\sigma_{X_{h} Y_{h}}^{2}=\sum_{\text {noth }}^{4} \sum_{v=0}^{\text {Msaxspeed }} X_{h} Y_{h} p_{v n}-\mu_{X_{h}} \mu_{Y_{h}}
$$

$$
\eta\left(S_{X_{h}}, S_{Y_{h}}\right)=\frac{\left(s_{X_{h}}-\mu_{X_{X_{h}}}\right)^{2}}{\sigma_{S_{X_{h}}}^{2}}+\frac{\left(s_{Y_{h}}-\mu_{S_{Y_{h}}}\right)^{2}}{\sigma_{S_{Y_{h}}}^{2}}-\frac{2 p\left(s_{X_{h}}-\mu_{S_{X_{h}}}\right)\left(s_{Y_{h}}-\mu_{S_{Y_{h}}}\right)}{\sigma_{s_{X_{h}}} \sigma_{S_{Y_{h}}}}
$$

Evidently, harmonics of different orders have different statistic characteristics. In addition to the difference of magnitude of both real and imaginary parts for different order of harmonics, the distribution of phase angles for different harmonics are also different. For example, for fundamental component, the phasors are all located in the first quadrant in $x-y$ coordinate system, while for 3rd harmonics, some phasors may locate in second quadrant and for 5 th harmonics, phasors are distributed in all four quardants.

As the joint pdf is obtained, the pdf of the magnitude of the harmonics can then be calculated by applying polar coordinate transformation:

$$
f_{Z h}\left(Z_{h}\right)=\int_{0}^{2 \pi} z_{h} f_{S_{X h} S_{Y h}}\left(z_{h} \cos \theta, z_{h} \sin \theta\right) d \theta
$$

The characteristics of the sum of current harmonics and the Total Harmonics Distortion are investigated using equation (13) (17) for 8 trains whose behavior states are assumed to be independent from each other and have the same speed PDF of Fig. 4. The PDF of fundamental and harmonics components are plotted in Fig. 6,7 respectively. The dots are the probability density function by Monte Carlo simulation while the solid lines are the PDF by calculated by equations (13) (17). It can be seen the two methods agree with each other very well.

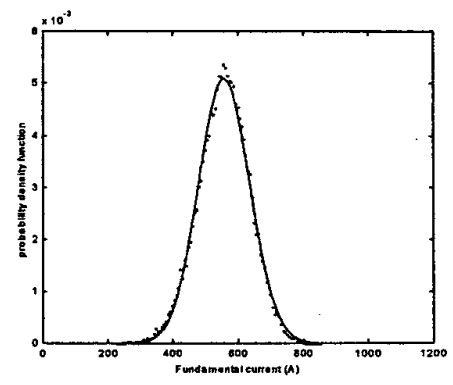

a) fundamental component

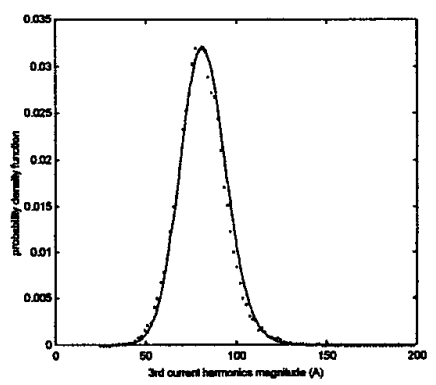

b) $3^{\text {rd }}$ component

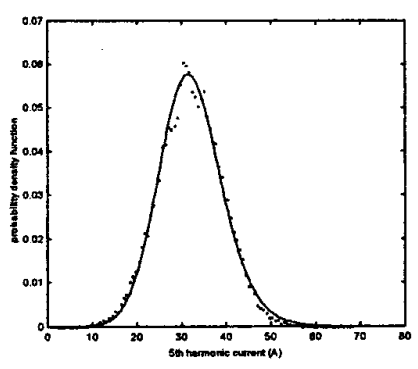

c) $5^{\text {th }}$ component

Fi g. 6. The probability density function of fundamental, 3rd and 5 th components for a system with 8 trains by analytic methods (dotted line is by simulation) 


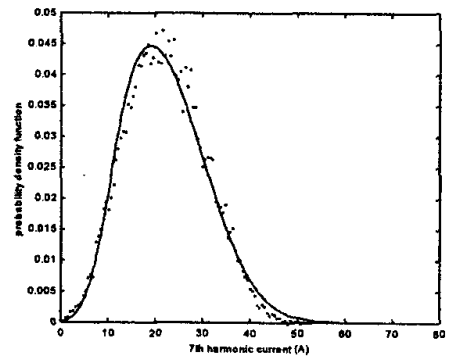

a) $7^{\text {th }}$ component

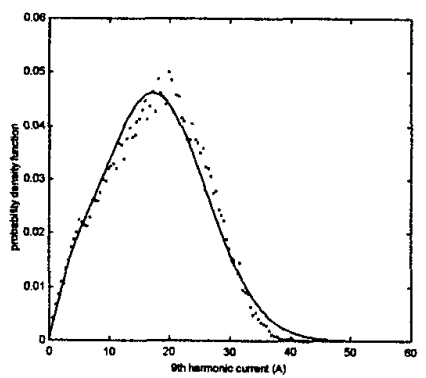

b) $g^{\text {th }}$ component

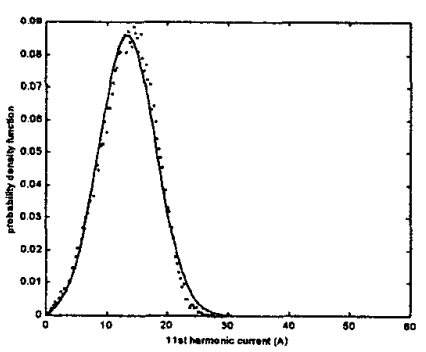

c) $11^{\text {st }}$ component

Fig. 7. The probability density function of the sum current harmonics of order 7 th, 9 th and 11 st respectively for a system with 8 trains by analytic methods (dotted line is by simulation)

The correlation coefficients for different harmonic orders are plotted in Fig. 8. The correlation coefficients do not change with the number of trains, but for different harmonics, the values may be different. The harmonics per train is plotted in Fig. 9 against train nu mber. Harmonics per train for multitrain system is lower than that for single train and this is a result of the harmonics cancellation. From the figure it can also be seen that as the number of trains increases, the harmonics per train decreases, which indicate that the opportunity for harmonics cancellation is higher if the number of harmonics sources increases. However, this tendency become very flat as the train number is greater than a certain value. For example, for the 9th harmonics, this number is 7 .

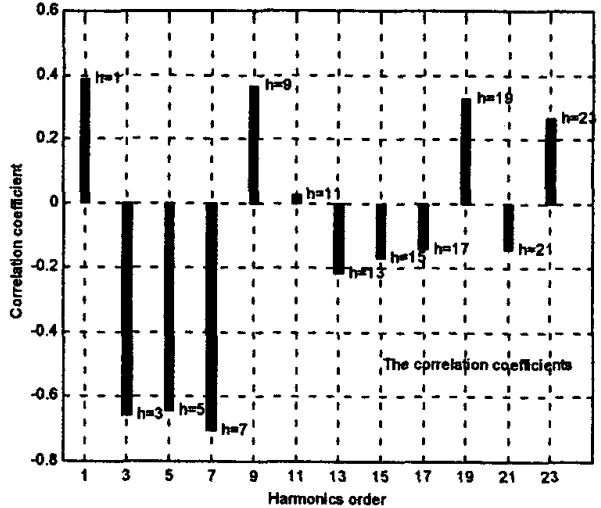

Fig. 8. The correlation coefficient for different harmonics

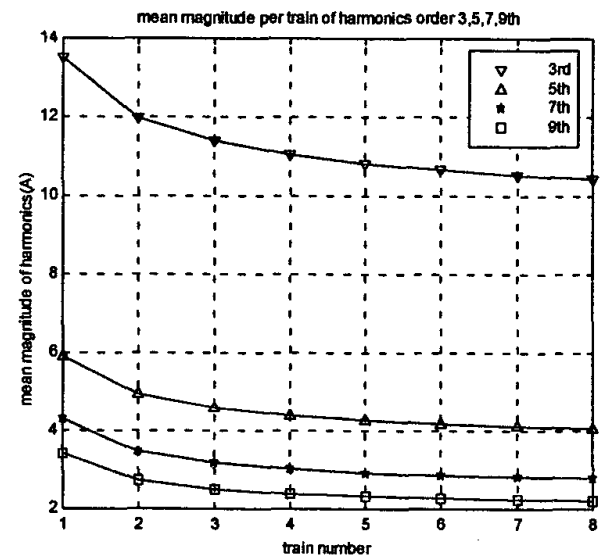

Fig. 9. The 3rd, 5th, 7th, 9th harmonics per train versus train number

\section{A COMPARISON OF THE THREE METHODS}

As a comparison between the proposed method and Monte Carlo simulation, we define the error between the two methods as:

$$
e r r_{h}=\sqrt{\int_{0}^{\max }\left(p_{a}\left(i_{h}\right)-p_{s}\left(i_{h}\right)\right)^{2} p_{s}\left(i_{h}\right) d i_{h}}
$$

where the $P_{a}$ is the probability density function by analytic method and $P_{5}$ is the probability density function by Monte Carlo simulation which is regarded as benchmark value in this comparison.

If the two methods match well, the error will be small, otherwise it will be large. This error also reflects the error over the whole scope, with emphasis on the central area. In Table V, the error for different number of trains and different harmonics orders are given. It can be seen, the error for the case of large number of trains is very small and the 
performance for low order harmonics is better than that for high order harmonics.

Table $V$ The error for different harmonics \& train numbers

\begin{tabular}{|c|c|c|c|c|c|c|c|c|}
\hline & $\mathrm{N}=1$ & $\mathrm{~N}=2$ & $\mathrm{~N}=3$ & $\mathrm{~N}=4$ & $\mathrm{~N}=5$ & $\mathrm{~N}=6$ & $\mathrm{~N}=7$ & $\mathrm{~N}=8$ \\
\hline $\mathrm{h}=1$ & 0.16 & 0.01 & 0.18 & 0.13 & 0.11 & 0.12 & 0.11 & 0 \\
\hline $\mathrm{h}=3$ & 0.35 & 0.1 & 0.27 & 0.11 & 0.07 & 0.04 & 0.03 & 0.01 \\
\hline $\mathrm{h}=5$ & 2.06 & 0.33 & 1.24 & 0.85 & 0.62 & 0.59 & 0.48 & 0.01 \\
\hline $\mathrm{h}=7$ & 3.29 & 0.45 & 1.76 & 1.01 & 0.82 & 0.7 & 0.65 & 0.02 \\
\hline $\mathrm{h}=9$ & 4.12 & 0.5 & 4.49 & 3.36 & 2.52 & 1.98 & 2.02 & 0.02 \\
\hline $\mathrm{h}=11$ & 6.33 & 0.46 & 0.75 & 0.29 & 0.24 & 0.22 & 0.22 & 0.03 \\
\hline $\mathrm{h}=13$ & 6.03 & 1.54 & 1.76 & 0.72 & 0.43 & 0.39 & 0.35 & 0.07 \\
\hline
\end{tabular}

Comparison of Monte Carlo method, proposed method and experimental method for the harmonics investigation of the traction system is presented in Table VII. The experiment method based on statistical results of harmonics on the harmonic current samples which are obtained directly from the trains in service. Large number of reliable samples are necessary for accurate evaluation of the system harmonics, thus this method is expensive and sometimes difficult to arrange. While for the proposed method, only samples of speed are necessary, which can be easily measured compared with current harmonics. For some systems, this can even be obtained directly from the schedule or control center of the traction system. To get the data for modeling of the electrical unit of the train, measurement can be arranged on an out of service train, rather than a train in service. The proposed method has almost the same accuracy as that of the Monte Carlo simulation method. Therefore this approach is a good choice for railway traction system harmonics evaluation without sacrificing accuracy of the prediction.

Table VI The comparison among three approaches

\begin{tabular}{|c|c|c|c|}
\hline method & experiment & simulation & proposed \\
\hline $\begin{array}{c}\text { total time } \\
\text { expected }\end{array}$ & months & $\begin{array}{c}1 \text { week in Pentium } \\
\text { II }\end{array}$ & 1 hour \\
\hline cost & expensive & not expensive & cheap \\
\hline $\begin{array}{c}\text { preparation } \\
\text { work }\end{array}$ & $\begin{array}{c}\text { data logging } \\
\text { system design, } \\
\text { programming }\end{array}$ & $\begin{array}{c}\text { modeling and pdf } \\
\text { of random } \\
\text { variables }\end{array}$ & $\begin{array}{c}\text { modeling and } \\
\text { pdf of random } \\
\text { variables }\end{array}$ \\
\hline accuracy & accurate & high & good \\
\hline
\end{tabular}

\section{CONCLUSION}

Probabilistic investigation of current harmonics of a local railway system in Hong Kong is presented. With the knowledge of the probability density function (p. d. f.) of speed and notch of trains, the mean and variance of current harmonics of a single traction electrical unit can be computed by analytic approach provided the simulation model or mathematical model of the traction electrical unit can be established. Based on the Large Number Law and Central Limit Theorem, the vectorial summation of current harmonics of a number of trains will follow normal distribution, thus the harmonics can be evaluated as long as the mean and variance known. The results obtained from the proposed method are in good accordance with Monte Carlo simulation. Parts of the results are also verified by experimental data. It is demonstrated harmonics analysis of traction system with random loading can be performed in this way, with no sacrifice of the accuracy. This method can be easily expanded to other cases.

\section{ACKNOWLEDGMENTS}

The authors would like to acknowledge Mr. Tony Lee, Rolling Stock Design Manger, Mr. W.C. Lam, High voltage Engineering Manager and their colleagues from East Rail Division, Kowloon-Canton Railway Corporation for their help and arrangement of our tests and measurement.

\section{REFERENCES}

J. A . Orr, A. E. Emanuel, K. W. Oberg, Current harmonics generated by a cluster of electric vehicle battery chargers, IEEE Trans. Power System 101, No.3 March 1982, pp691700.

D. Okelly, Probability characteristics of fundamental and harmnic sequence components of randomly varying loads, IEE-PROC., Vol. 129, Pt. C. No.2, March 1982, pp70-78.

R.E.Morrison, A. D. Clark, Probabilistic representation of harmonic currents in AC traction system, IEE PROC., Vol.131. Pt. B, No.5, Sept. 1984, pp181-189.

A. E. Emanuel, S. R. Kaprielian, Contribution to the theory of stochastically periodic harmonics in power systems, IEEE Trans. Power Delivery, Vol. PWRD-1, No. 3, July 1986, pp285-293.

Y. Baghzouz, Owen Tan, Probabilistic modeling of power system harmonics, IEEE Trans. Ind. Application, Vol. 23, No. 1, Jan. 1987, pp 173-180.

W. Kazibwe, et al, IEEE Trans. Power Delivery, Vol.4, No. 1, Jan. 1989, pp621-628.

Y. J. Wang, Lambert Pierrat, Probabilistic modeling of current harmonics produced by an AC/DC converter under voltage unbalance, IEEE Trans. Power Delivery, Vol. 8, No.4, 1994, pp2060-2066.

Y. J. Wang, Lambert Pierrat, Summation of harmonic currents produced by AC/DC static converters with randomly fluctuating loads, IEEE Trans. Power Delivery, Vol. 9, No.2, April 1994, pp1129-1135.

S.R. Kaprielioan, A.E. Emanuel, R. Dwyer, H. Melita, Predicting voltage distortion in a system with multiple random harmonic sources, IEEE Trans. Power Delivery, Vol. 9, No.3, July 1994, pp1632-1638.

P. Staats, W. Grady, A. Arapostathis, R. Thallam, A statistical method for predicting the net harmonic currents generated by a concentration of electric vehicle battery chargers, IEEE Trans. Power Delivery, Vol.12, No.3, July 1997, pp1258-1266

K. H. Yuen, M. H. Pong, W. C. Lo, Z. M. Ye, Modeling of electric railway vehile for harmonic analysis of traction power supply system using spline interpolation in frequency domain, IEEE APEC 1999.

Ye Zhongming, M. H. Pong, W. C. Lo, Yuen K. H., Harmonic evaluation of traction system by Monte Carlo simulation, IEEE PESC 1999.

R. Kadhim, J. Allan, B. Mellitt, Assessment of AC side harmonics of DC supplied railways, EPE conference proceeding, pp 256-261. 